\title{
ON THE PRIMITIVITY OF GROUP RINGS OF AMALGAMATED FREE PRODUCTS
}

\author{
BOLA O. BALOGUN
}

(Communicated by Donald S. Passman)

\begin{abstract}
In this paper we prove the primitivity of the group ring $F[G]$ where $F$ is a field and $G$ belongs to a certain class of free products of groups with amalgamation studied recently by the author. Thus our result generalizes a result of Formanek on free products of groups.
\end{abstract}

\section{INTRODUCTION}

Let $\mathbf{V}$ denote the class of groups which satisfy the following conditions (a), (b) and (c):

(a) $G=(A * B ; K=\varphi(H))$ is the free product of two groups $A$ and $B$ with the subgroup $H$ and $K$ amalgamated.

(b) $H$ does not contain the verbal subgroup $A\left(X^{2}\right)$ of $A$ and $K$ does not contain the verbal subgroup $B\left(X^{2}\right)$ of $B$.

Consequently, $G$ contains a copy of $F_{2}$ freely generated by $x=a b a$ and $y=b a b$, where $a^{2} \notin H$ and $b^{2} \notin K$ ([1], Lemma 3.2).

(c) $\left(a^{-1} H a\right) \cap H=\{e\}=\left(b^{-1} K b\right) \cap K$.

For example, if

$$
A=\left\langle a, c ; a^{3}, c^{2},(a c)^{2}\right\rangle \text { and } B=\left\langle b, d ; b^{3}, d^{2},(b d)^{2}\right\rangle,
$$

the symmetric group on three objects, then the free product of $A$ and $B$ with the cyclic groups generated by $c$ and $d$ amalgamated is a group in $\mathbf{V}$.

Let $F$ be a field, $G$ a group and $F[G]$ the group ring of $G$ over $F$. If $G=\mathbf{Z} * \mathbf{Z}$ or $G_{1} * G_{2}$, where $\mathrm{Z}$ is the infinite cyclic group and $G_{1}, G_{2}$ are not both of order 2 , then it is known that $F[G]$ is primitive $[4,2]$. In this paper, we show that $F[G]$ is primitive if $G$ is in $\mathbf{V}$, thus generalizing the results of [4 and 2], except when $G=G_{1} * G_{2}$ where $G_{1}$ or $G_{2}$ has exponent 2 .

\section{NOTATION AND DEFINITIONS}

Let $G=(A * B ; K=\varphi(H))$. Suppose specific right coset representative systems for $A(\bmod H)$ and $B(\bmod K)$ have been selected. Then any element $g$ in

Received by the editors July 25, 1988 and, in revised form, October 11, 1988.

1980 Mathematics Subject Classification (1985 Revision). Primary 16A27, 20 C07.

Key words and phrases. Group rings, amalgamated free products. 
$G$ can be represented uniquely as a product $h c_{1} c_{2} \cdots c_{r}$ called the reduced form of $g$, where $h$ is in $H, c_{i}$ is not in $H, c_{i}$ is a representative from $A(\bmod H)$ or $B(\bmod K)$ and $c_{i}, c_{i+1}$ are not both in $A$ or both in $B$. The non-negative integer $r$ is called the (representative) length $l(g)$ of $g$. If $g=h c_{1} c_{2} \cdots c_{r}$ is in the reduced form, then $g$ is said to begin with $c_{1}$ and end with $c_{r}$. If $c_{1}$ is in $A$, we say that $g$ has $A$-form. We define $-A,-B$, and $B$ - forms for elements of $G$ similarly. If $c_{1}$ is in $A$ and $c_{r}$ is in $B$, we say that $g$ has $A B$ form. We define $A A, B B$, and $B A$ forms similarly.

An ideal $I$ in the ring $R$ is called (right) primitive if it is the largest ideal contained in some maximal right ideal $M$, thus

$$
I=R^{\cdot} \cdot M=\{r \in R \mid R r \subset M\} .
$$

This definition is not symmetrical, and it is known that right primitivity does not imply left primitivity. Nonetheless, the attribute "right" is usually omitted. A ring is called (right) primitive if 0 is a primitive ideal.

A module $A_{R}$ is called faithful if, for any $0 \neq r \in \mathbf{R}, A r \neq 0$. The following characterization of primitive rings is often taken as their definition.

Theorem (Jacobson). The ring $R$ is primitive if and only if there exists a faithful irreducible module $A_{R}$.

Proof. See [3, p. 52].

\section{Primitivity}

Theorem 3.1. Let $G$ be a group in $\mathbf{V}$, and let $R$ be an $F$-algebra. If $R$ has no zero divisors and if $|R| \leq|G|$, an inequality of possibly infinite cardinals, then $R[G]$ is a primitive ring.

Proof. For definiteness, we assume that $|A| \geq|B|$. By the defining condition (b) of $\mathbf{V}, G$ contains a copy of $F_{2}$ and so it is infinite and its cardinality is not less than that of $R$, hence $G$ and $R[G]$ must have the same cardinality. The proof is broken into two cases, depending on the finiteness or otherwise of $[A: H]$.

Case 1. The index of $H$ in $A$ is infinite.

Since $A$ is infinite, $|A| \geq|B|$ and $|A * B| \geq\left|A *{ }_{H} B\right|$, it is clear that $|A|=|G|$. Hence since $|G| \geq|R|$, we have $|A|=|R[G]|$. Let $\alpha: u \mapsto \alpha(u)$ be a fixed bijection from $A$ onto $R[G]$, such that $\alpha(1)=0$.

Let $a, b, x, y$ be as in $\S 2$, and $w(u)$ an element of maximal length in the support of $\alpha(u)$, if $u \neq 1$. As in [2] we now construct a suitable linear form in $\alpha(u)$ that exhibits the element $u$ in its structure. 
For each $u \in H-\{1\}$, let $m(u)$ equal

$$
\begin{array}{ll}
a u \alpha(u) a^{-1} b+b a u \alpha(u) a^{-1}+1 & \text { if } w(u) \in H \\
u a b \alpha(u) b+u y \alpha(u)+1 & \text { if } w(u) \text { is of } A A \text { form } \\
u y \alpha(u)+u a b \alpha(u)+1 & \text { if } w(u) \text { is of } A B \text { form } \\
u a \alpha(u) b+u b a \alpha(u)+1 & \text { if } w(u) \text { is of } B A \text { form } \\
u b a \alpha(u)+u a \alpha(u) a+1 & \text { if } w(u) \text { is of } B B \text { form }
\end{array}
$$

For each $u \in A-H$, let $m(u)$ equal

$$
\begin{array}{ll}
u \alpha(u) b+b u \alpha(u)+1 & \text { if } w(u)=1 \\
u b a \alpha(u) a^{-1} b+b u b \alpha(u)+1 & \text { if } w(u) \in H-\{1\} \\
u b \alpha(u) b+b u b \alpha(u)+1 & \text { if } w(u) \text { is of } A A \text { form } \\
b u b \alpha(u)+u b \alpha(u)+1 & \text { if } w(u) \text { is of } A B \text { form } \\
u \alpha(u) b+b u \alpha(u)+1 & \text { if } w(u) \text { is of } B A \text { form } \\
b u \alpha(u)+u \alpha(u) u+1 & \text { if } w(u) \text { is of } B B \text { form }
\end{array}
$$

We note that

(i) Every maximal length element in the support of $m(u)$ is of form $A$ - or $B A-$.

(ii) There exist maximal length elements in the support of $m(u)$ which have $-A$ form and which have $-B$ form.

Let $s(u)$ be some nonzero element of $R[G]$, and $w_{0}$ an element of maximal length in its support. By (ii) there exists an element $t_{0}$ of maximal length in the support of $m(u)$ such that the product $t_{0} w_{0}$ is in reduced form. Since $R$ is a ring with no zero divisors, $t_{0} w_{0}$ is contained in the support of $m(u) s(u)$. Hence we can deduce from (i) that

(iii) If $s(u) \neq 0$, then every maximal length element in the support of $m(u) s(u)$ has form $A$ - or $B A$ - .

Hence the right ideal in $R[G]$ generated by $\{m(u): u \in A-\{1\}\}$ is proper. For otherwise we would get an equation of the form

$$
1=\sum_{j=1}^{k} m\left(u_{j}\right) s\left(u_{j}\right)
$$

for some $s\left(u_{j}\right)(j=1,2, \ldots, k)$, which contradicts (iii). Therefore, this right ideal is proper and so it is contained in a maximal ideal $M$. Consider the irreducible module $W=R[G] / M$. Let $\rho$ be a nonzero element of $R[G]$ such that $I=R[G] \rho \subset M$. Since $I \neq 0$, it contains the element $\alpha(u)$ for some $u \in A-\{1\}$ and so by the definition of $\alpha(u), \alpha(u)-1 \in I \subset M$. Hence $1 \in M$, contrary to the choice of $M$ as a maximal (proper) ideal. Therefore $R[G]^{\circ} \cdot M=0$, and $W$ is faithful. Thus $R[G]$ is a primitive ring.

Case 2. The index of $H$ in $A$ is finite. 
Since $|A: H| \geq|B: K|$, it is clear that both $|G: H|$ and $|R[G]: R[H]|$ are countably infinite. Let $\{\alpha(n): n=1,2,3, \ldots$,$\} be an enumeration of a right$ coset representative system for $R[G](\bmod R[H])$. Let $a$ and $b$ be as before so that $a^{2} \notin H$ and $b^{2} \notin K$. Let $w(n)$ be a group element of maximal length in the support of $\alpha(n)$ and we let $m(n)$ equal

$$
\begin{array}{ll}
(a b)^{n} a^{2} \alpha(n) b+b(a b)^{n} a^{2} \alpha(n)+1 & \text { if } w(n)=1 \text { or } w(n) \text { is of } B A \text { form } \\
(a b)^{n} a^{2} b a \alpha(n) a^{-1} b+b(a b)^{n} a^{2} b \alpha(n)+1 & \text { if } w(n) \in H-\{1\} \\
(a b)^{n} a^{2} \alpha(n) a+b(a b)^{n} a^{2} \alpha(n)+1 & \text { if } w(n) \text { is of } B B \text { form } \\
(a b)^{n} a^{2} b \alpha(n) b+b(a b)^{n} a^{2} b \alpha(n)+1 & \text { if } w(n) \text { is of } A A \text { form } \\
(a b)^{n} a^{2} b \alpha(n) a+b(a b)^{n} a^{2} b \alpha(n)+1 & \text { if } w(n) \text { is of } A B \text { form. }
\end{array}
$$

As in Case 1, we note firstly that every group element of maximal length in the support of $m(n)$ begins with $(a b)^{n} a^{2}$ or $b(a b)^{n} a^{2}$; secondly that there exist maximal length group elements in the support of $m(n)$ of the form $-A$ and of the form $-B$. From these we conclude as before that if $s(n) \neq 0$, then every maximal length group element in the support of $m(n) s(n)$ begins with either $(a b)^{n} a^{2}$ or $b(a b)^{n} a^{2}$. Thus $\Sigma_{n} m(n) R[G]$ is a proper ideal. This is extended to a maximal ideal $M$ which contains no nonzero ideal of $R[G]$. As before we can conclude that $R[G]$ is primitive. This completes the proof.

Theorem 3.2. Let $G \in \mathbf{V}$. If $F$ is any field, then $F[G]$ is a primitive ring. Proof. It suffices to show that

$$
\Delta(G)=\left\{g \in G:\left|G: C_{G}(g)\right|<\infty\right\}=\{1\} .
$$

Let $g \in G-\{1\}$, by Lemma 3.3 of [1] there exists an integer $k$ such that $(a b a)^{k} g(a b a)^{k}$ (when written in reduced form) begins and ends with a coset representative from $A(\bmod H)$. We can then alternately conjugate this new element by elements of $A-H$ and $B-K$ to obtain infinitely many conjugates of $g$. Thus $g \notin \Delta(G)$ and $\Delta(G)=1$.

Let $F$ be a given field and $F_{0}$ be its prime subfield. Then $F_{0}$ is countable, and so $\left|F_{0}\right| \leq|G|$. Taking $R=F_{0}$ in Theorem 3.1 , we have that $F_{0}[G]$ is primitive. Finally since $\Delta(G)=1$, the desired result follows by [ 5 or 6 , p. 356].

Remark 3.3. Let $S$ be a subgroup of a group $T$. A distinct pair $\left\{a_{1}, a_{2}\right\}$ of elements of $T$, neither of which are in $S$, is called a blocking pair for $S$ in $T$ if

(i) $a_{i}^{\varepsilon} a_{j}^{\delta} \notin S, \quad 1 \leq i, j \leq 2, \varepsilon= \pm 1, l \delta= \pm 1$, unless $a_{i}^{\varepsilon} a_{j}^{\delta}=e$, and

(ii) if $x \in S, \quad x \neq e$, then

$$
a_{i}^{\varepsilon} x a_{j}^{\delta} \notin S, \quad 1 \leq i, j \leq 2, \varepsilon= \pm 1, \delta= \pm 1 .
$$


Let $G=A *_{H} B$ where $A \neq H \neq B$ and there exists a blocking pair $\left\{x_{1}, x_{2}\right\}$ for $H$ in one of the factors of $G$, then it can be shown that $F[G]$ is primitive for any field $F$.

\section{REFERENCES}

1. B. O. Balogun, $C^{*}$-algebras associated with amalgamated product of groups, Glasgow Math. J. 29 (1987), 143-148.

2. E. Formanek, Group rings of free products are primitive, J. Algebra 26 (1973), 508-511.

3. J. Lambek, Lectures on rings and modules, Blaisdell Publishing Co., Mass., 1966.

4. C. McGregor, On the primitivity of the group ring of a free group, Bull. London Math. Soc. 8 (1976), 294-298.

5. D. S. Passman, Primitive group rings, Pacific J. Math. 47 (1973), 499-506.

6. __ The algebraic structure of group rings, John Wiley \& Sons, New York, 1977.

Mathematics Department, Obafemi Awolowo University, Ile-Ife, Nigeria 